\title{
Bronchoalveolar lavage-detected SARS-CoV-2 infection in presence of serial negative nasopharyngeal swabs: a case report
}

\author{
Grazia Caci ${ }^{1} \wedge$, Fabrizio Minervini ${ }^{2}$, Carsten Fechner ${ }^{3}$, Justus E. Roos ${ }^{3}$, Ellen C. Obermann ${ }^{4}$, \\ Andrea Azzola ${ }^{5}$
}

${ }^{1}$ Department of internal Medicine, Lucerne Cantonal Hospital, Lucerne, Switzerland; ${ }^{2}$ Department of Thoracic Surgery, Lucerne Cantonal Hospital, Lucerne, Switzerland; ${ }^{3}$ Department of Radiology and Nuclearmedicine, Lucerne Cantonal Hospital, Lucerne, Switzerland; ${ }^{4}$ Pathology, Lucerne Cantonal Hospital, Lucerne, Switzerland and University of Basel, Basel, Switzerland; ${ }^{5}$ Department of internal Medicine, Service of Pulmonology, Lucerne Cantonal Hospital, Lucerne, Switzerland

Correspondence to: Dr. Grazia Caci, MD. Department of Internal Medicine, Luzerner Kantonsspital, CH-6000 Luzern 16, Switzerland.

Email: grazia.caci15@gmail.com.

\begin{abstract}
We describe a case of a SARS coronavirus 2 (SARS-CoV-2) infection in a Swiss 54-years-old immunocompromised patient (lymphoma, therapy with the anti-CD20 antibody Rituximab ${ }^{\circledR}$ ), with initial scarce respiratory symptoms but typical coronavirus disease 2019 (COVID-19) radiological presentation, and symptoms onset during a holiday trip to Texas (USA). Three nasopharyngeal swabs in the 96 hours following hospital admission were negative, despite a CT thorax suggestive for an early stage of infection. COVID-infection was finally confirmed in the bronchoalveolar lavage (BAL) fluid, performed for exclusion of an alternative diagnosis in immunocompromised. In the BAL an increased cellularity with marked lymphocytosis of $35 \%$, a reduced CD4/CD8 ratio of 0.1 and borderline neutrophilia of $3 \%$ were found. This finding might be due to the concomitant therapy with anti-CD20 antibodies, but the presence of lymphocytosis in the BAL despite peripheral lymphopenia with decreased CD4/CD8 T-cells ratio are described here for the first time in a SARS-CoV-2 infection. Persistent gastrointestinal symptoms (diarrhea), fever and initially headache were the predominant symptoms. The respiratory symptoms were scarce (variable mild dyspnea mMRC1). The respiratory conditions worsened during the hospital stay, with tachypnea up to $35 / \mathrm{min}$, increased need for supplemental oxygen up to $8 \mathrm{~L} / \mathrm{min}$ and worsening lung infiltrates on CT thorax on day 5. A therapy with hydroxychloroquine (HCQ) and an immunoglobulin-supplementation were given, with clinical and respiratory improvement, without need for intensive care or any ventilator support, and hospital discharge on day 16. Our case highlights some diagnostic and therapeutical challenges occurring in patients with COVID-19 infection. As take-home message, in the presence of clinical and radiological findings compatible with SARS-CoV-2 infection we outline the importance of treating patients accordingly, also in presence of repeated negative nasopharyngeal swabs. In selected patients as in our case a bronchoscopic BAL should be considered to exclude other infections, but in our opinion not primarily to confirming COVID-19 infection. Our unique finding of a lymphocytosis in the BAL during a COVID-19 infection needs further investigations.
\end{abstract}

Keywords: SARS coronavirus 2 (SARS-CoV-2); coronavirus disease 2019 (COVID-19); swab; bronchoalveolar lavage (BAL); lymphocytosis

Submitted May 27, 2020. Accepted for publication Feb 04, 2021.

doi: $10.21037 /$ atm-20-4307

View this article at: http://dx.doi.org/10.21037/atm-20-4307

^ ORCID: 0000-0003-1325-0466. 


\section{Introduction}

In December 2019 a new pattern of atypical interstitial pneumonia was reported for the first time in Wuhan, Hubei, China (1). SARS coronavirus 2 (SARS-CoV-2), a positive-sense single stranded RNA Virus, has been identified as the causative agent of coronavirus disease 2019 (COVID-19). The human to human transmission of the virus caused a rapid progressing global pandemia. More common reported symptoms were fever, cough, fatigue, anosmia, ageusia, nasal congestion, while palpitation, diarrhea and myalgia are quite rare. The lung involvement with progressive respiratory insufficiency is usually responsible for the severe course of the disease, with ARDS and high morbidity and mortality, specially in polymorbide or elderly patients. What turns out to be unique in our case are the initial potentially misleading clinical presentation with gastrointestinal symptoms and fever in the absence of respiratory symptoms despite suspicious radiology findings, the several and repeated negative nasopharyngeal swabs for SARS-CoV-2 which could have led to the thought of a Hospital acquired pneumonia (HAP) but a positive bronchoscopic bronchoalveolar lavage (BAL) positivity for COVID-19, and the finding of a marked lymphocytosis in the BAL. We present the following article in accordance with the CARE reporting checklist (available at http:// dx.doi.org/10.21037/atm-20-4307).

\section{Case presentation}

A 54-year-old Swiss man was admitted to our hospital on March 22th because of unspecific variable symptoms in the last two weeks, with an onset during a holiday trip in Texas (USA). He had already been known for a follicular lymphoma WHO 1-2 Stage 4 first diagnosed in 2018 under therapy with the anti-CD20 monoclonal antibodies Rituximab (currently $700 \mathrm{mg}$ every 8 weeks, last administration on February 18th, 2020), without other co-morbidities. Before the trip, he was asymptomatic. As soon as he arrived in the USA on March 6th he suffered from gastric discomfort with diarrhea up to 4-5 times a day. Subsequently, he developed bifrontal position-dependent headache (visual analog pain scale 5-6/10) causing sleeping difficulties. After his return to Switzerland on March 19th, 2020, he developed an undirected dizziness. He never measured fever before hospital admission. Cough and rhinitis were denied. Only a mild variable dyspnea NYHA I was reported.

A timetable of our case is shown on Figure 1.
On admission he presented with a core temperature of $38.2{ }^{\circ} \mathrm{C}$, a blood pressure of $130 / 90 \mathrm{mmHg}$, a heart rate of $110 \mathrm{bpm}$, a respiratory rate of 15 breaths/min and an oxygen saturation of $90 \%$ at room air. The laboratory results showed a white blood cell count of $3.3 \mathrm{G} / \mathrm{L}$ with lymphopenia of $0.62 \mathrm{G} / \mathrm{L}$; kidney function and pancreatic and liver enzymes were normal, excepted an LDH of $592 \mathrm{U} / \mathrm{L}$. The blood sugar was slightly elevated at $6.4 \mathrm{mmol} / \mathrm{L}$, and the sodium slightly reduced at $131 \mathrm{mmol} / \mathrm{L}$. The C-reactive protein level was $39 \mathrm{mg} / \mathrm{L}$, the arterial blood gas analysis showed a $\mathrm{pH}$ of $7.50, \mathrm{PaCO}_{2} 3.78 \mathrm{KPa}, \mathrm{PaO}_{2} 10.2 \mathrm{KPa}$, bicarbonate $22 \mathrm{mmol} / \mathrm{L}$, base excess $1.10 \mathrm{mmol} / \mathrm{L}$, lactate $0.8 \mathrm{mmol} / \mathrm{L}$. Contrast head computed tomography (CT) excluded bleeding, sinus vein thrombosis and signs of endocranial hypertension. In absence of meningeal signs, rachicentesis was not performed.

The chest X-ray, compared to a previous image made 4 weeks before, showed new bilateral patchy reticular opacities with predominant involvement of the lower lobes and without pleural effusions compatible with atypical pneumonia (Figure 2). The CT thorax confirmed the diagnosis. Bilateral mostly peripheral but also peribronchovascular ground-glass opacities with basal predominant distribution were seen, well compatible with an atypical pneumonia of viral origin. These findings were classic for an early stage SARS-CoV-2 infection judged by the new British Society of Thoracic Imaging (BSTI) guidelines (2). The total severity score of the involved lung parenchyma was 7 of 20 (25-50\% of the lung parenchyma), therefore close to a critical ill patient collective (3). The differential diagnoses included other viral, bacterial or opportunistic infection and pharmacological toxicity, which were considered unlikely.

The patient was isolated, and antibiotics were initially prescribed (clarithromycin and ceftriaxone). The first oropharyngeal swab for COVID-19, which was taken on admission, was negative, as well as a second swab 24 hours later. Initial multiplex-PCR swabs for respiratory viral infections was negative. In the 72 hours following hospital admission no improvement of symptoms occurred. The patient remained febrile up to $39^{\circ} \mathrm{C}$, variable mild dyspnea and diarrhea persisted, and an increasing oxygen need of up to $6 \mathrm{lpm}$ to maintain $\mathrm{SpO}_{2}$ above $90 \%$ was observed. At this point, D-dimers and ferritin were $863 \mathrm{ng} / \mathrm{mL}$ and 2,869 $\mathrm{mcm} / \mathrm{L}$, respectively. The $\mathrm{LDH}$ values increased by up to $853 \mathrm{U} / \mathrm{L}$. PCR increased to $117 \mathrm{mg} / \mathrm{L}$.

Because of the underlying immunosuppression and the clinical worsening, although no pathogen was found in 


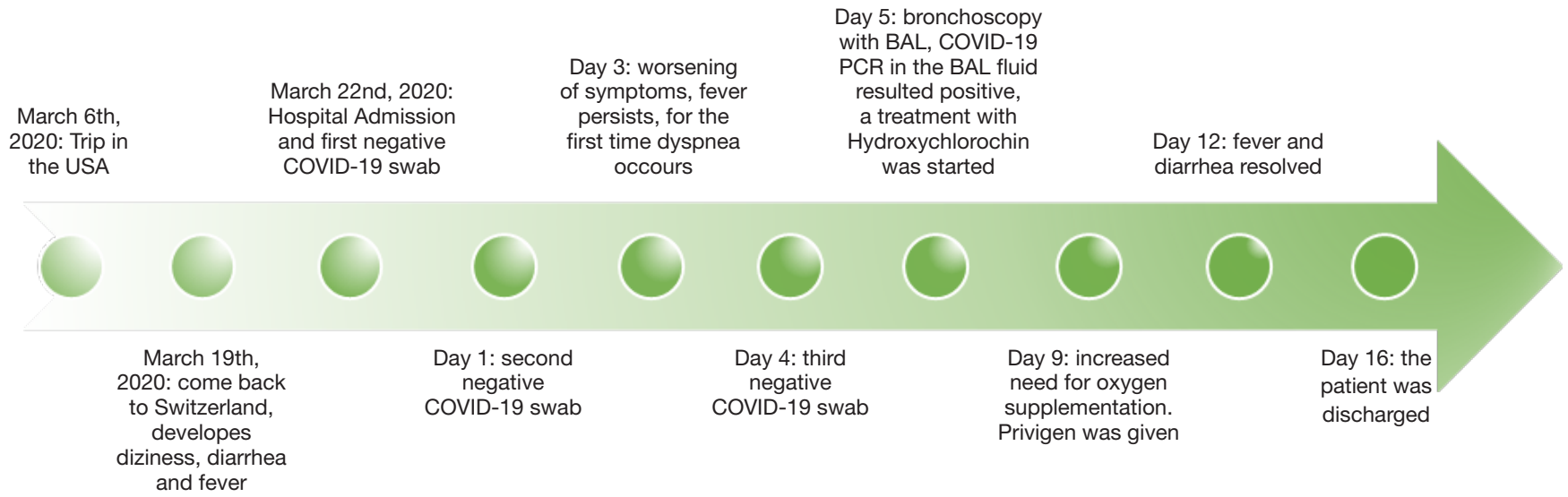

Figure 1 Timetable of the reported case. COVID-19, coronavirus disease 2019; BAL, bronchoalveolar lavage.
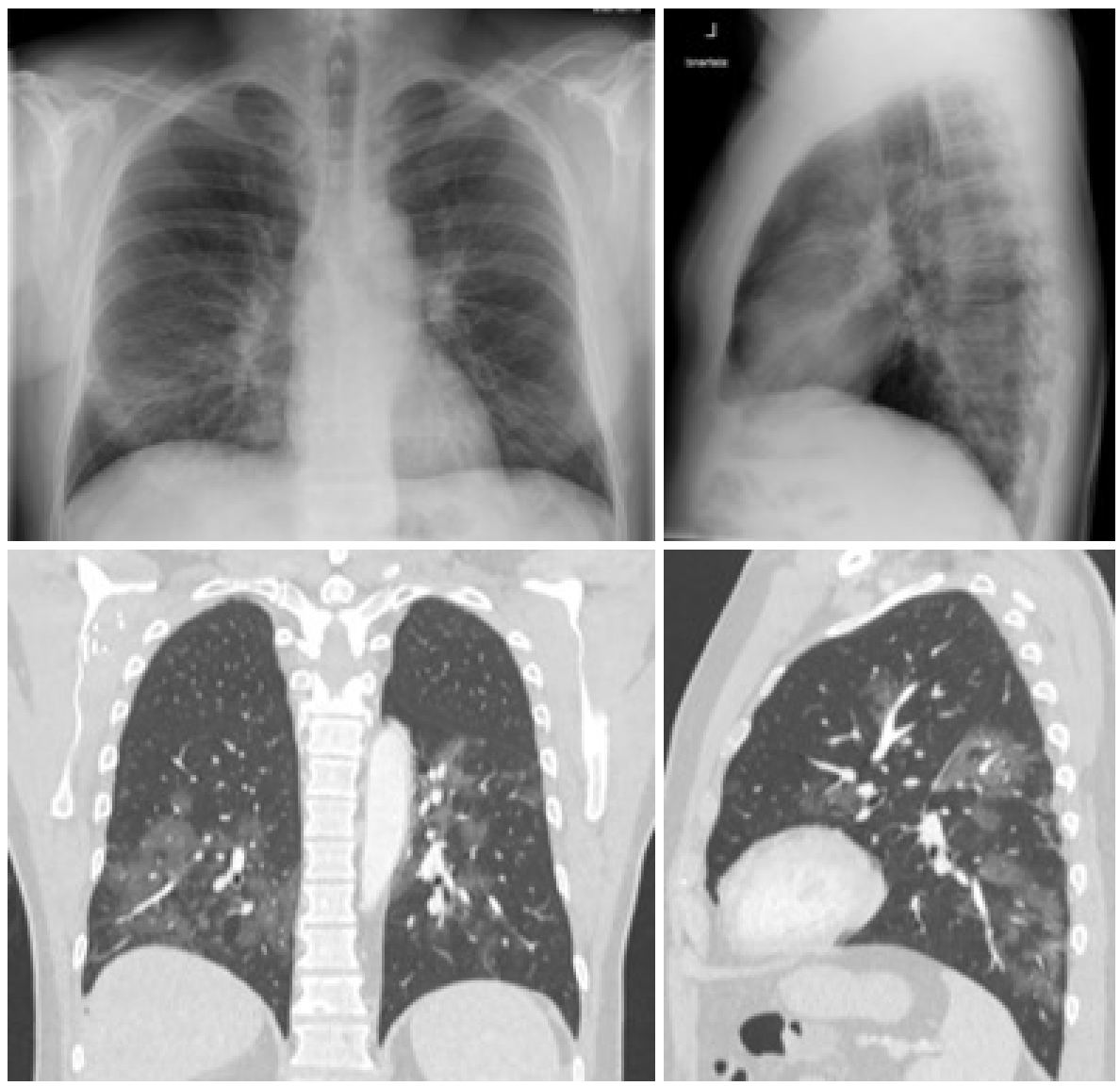

Figure 2 Comparison of Chest X-ray and CT-thorax at hospital admission, 10 days after onset of the first symptoms. Bilateral patchy reticular opacities with predominant involvement of the lower zones on the X-ray can be observed. Bilateral peripheral and peribronchovascular ground-glass opacities with basal predominant distribution in the CT-thorax are seen. 

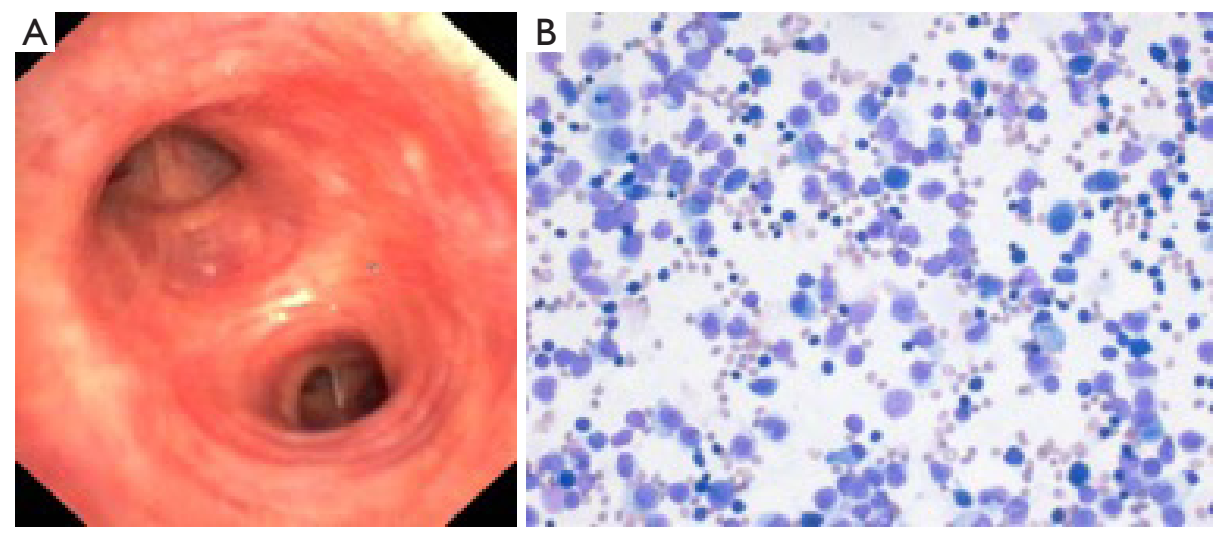

Figure 3 Endobronchial finding with cytological diagnostic of BAL specimens. (A) Endobronchial finding of an acute bronchitis in absence of secretions (upper left lobe); (B) lymphocytic alveolitis (May-Grunwald Giemsa stain; 20× magnification): Numerous lymphocytes are interspersed among macrophages. Of note, no changes suggestive of a viral infection or repair are present. BAL, bronchoalveolar lavage.

blood cultures, antibiotics were empirically changed to Piperazillin/Tazobactam, and due to the possible alternative diagnosis, the indication to BAL was given. Before BAL, a third COVID-19 nasopharyngeal swab was repeated almost 96 hours after hospital admission and confirmed negative.

The bronchoscopy showed a moderately inflamed bronchial mucosa in absence of bronchial secretions (Figure $3 A$ ). A BAL was performed in the middle lobe. After instillation of $150 \mathrm{~mL} 0.9 \%$ sterile saline solution, $80 \mathrm{~mL}$ of light turbid not hemorrhagic fluid was recovered. The differential count of the BAL (Figure 3B) revealed an increase of lymphocytes to $35 \%$, and a borderline increase of neutrophils to $3 \%$. Eosinophils were not increased. Immunocytochemical studies revealed a marked decrease of the CD4/CD8 ratio to 0.1 (CD4: clone 4B12, Novocastra, order no. NCL-L-CD4-368; CD8: clone C8/144B, BioSB, order no. BSB-BSB5173). CD20-positive B-lymphocytes were not present (clone L26, Agilent Technologies, order no. M075501). Careful reevaluation of the slides did not show any signs of a viral infection such as intranuclear or intracytoplasmic inclusion, nuclear enlargement or mutinucleation. Neither were there any signs of repair such as desquamated alveolar cells or nuclear atypia. Special stains did not reveal pneumocystis jirovecii or fungi (toluidin blue, Grocott). Broad microbiology investigations for viral, bacterial and fungal pathogens were negative, but the COVID-19 PCR in the BAL fluid resulted positive.

\section{Further management and evolution}

Following the BAL results, antibiotics were stopped and a therapy with hydroxychlorochin $200 \mathrm{mg}$ twice daily started. A follow-up CT confirmed the worsening of the pulmonary condition within 5 days. The total severity CT score increased to 14 of 20 (50-75\% of the lung parenchyma involved), clearly assigning the patient to the critical ill group. The initial ground glass got denser. Additional interlobular septal thickening with typical crazy paving was seen, representing alveolar edema and interstitial inflammation. Also, small areas of peripheral consolidations and small bilateral pleural effusion were observed (Figure 4). Hemodynamics remained stable but up to day 9 after admission the patient remained tachypneic and required an oxygen supplementation up to $8 \mathrm{~L} /$ min to maintain $\mathrm{SpO}_{2}$ above $88-90 \%$. The IL-6 level increased up to $136 \mathrm{pg} / \mathrm{mL}$, Ferritin up to $6,410 \mathrm{mcg} / \mathrm{L}$ and D-Dimer up to $1,146 \mathrm{ng} / \mathrm{mL}$. Due to the worsening conditions, the underlying disease, the Rituximab-therapy with complete CD-20 depletion and a slightly reduced total $\mathrm{IgG}$ level of $6.13 \mathrm{~g} / \mathrm{L}$ iv-human immunoglobulin substitution (Privigen ${ }^{\circledR} 30 \mathrm{~g}$ ) was given. Fever and diarrhea resolved on day 12 after hospital admission. Indication to ICU-transfer was reassessed on a daily basis according to the early-warning-score (4). Given the improvement of the general conditions and of the respiratory insufficiency ICU-treatment and/or noninvasive or mechanical ventilation were avoided. The patient was discharged on day 16 after admission, with improved general condition and a residual moderate respiratory partial insufficiency without indication to oxygen supplementation $\left(\mathrm{PaO}_{2} 8.92 \mathrm{kPa}, \mathrm{PaCO}_{2} 4.93 \mathrm{kPa}\right.$, $\mathrm{HCO}_{3-} 26.1 \mathrm{mmol} / \mathrm{L}, \mathrm{SaO}_{2} 94 \%$ at rest/room air).

A follow-up to exclude late consequences of COVID-19 

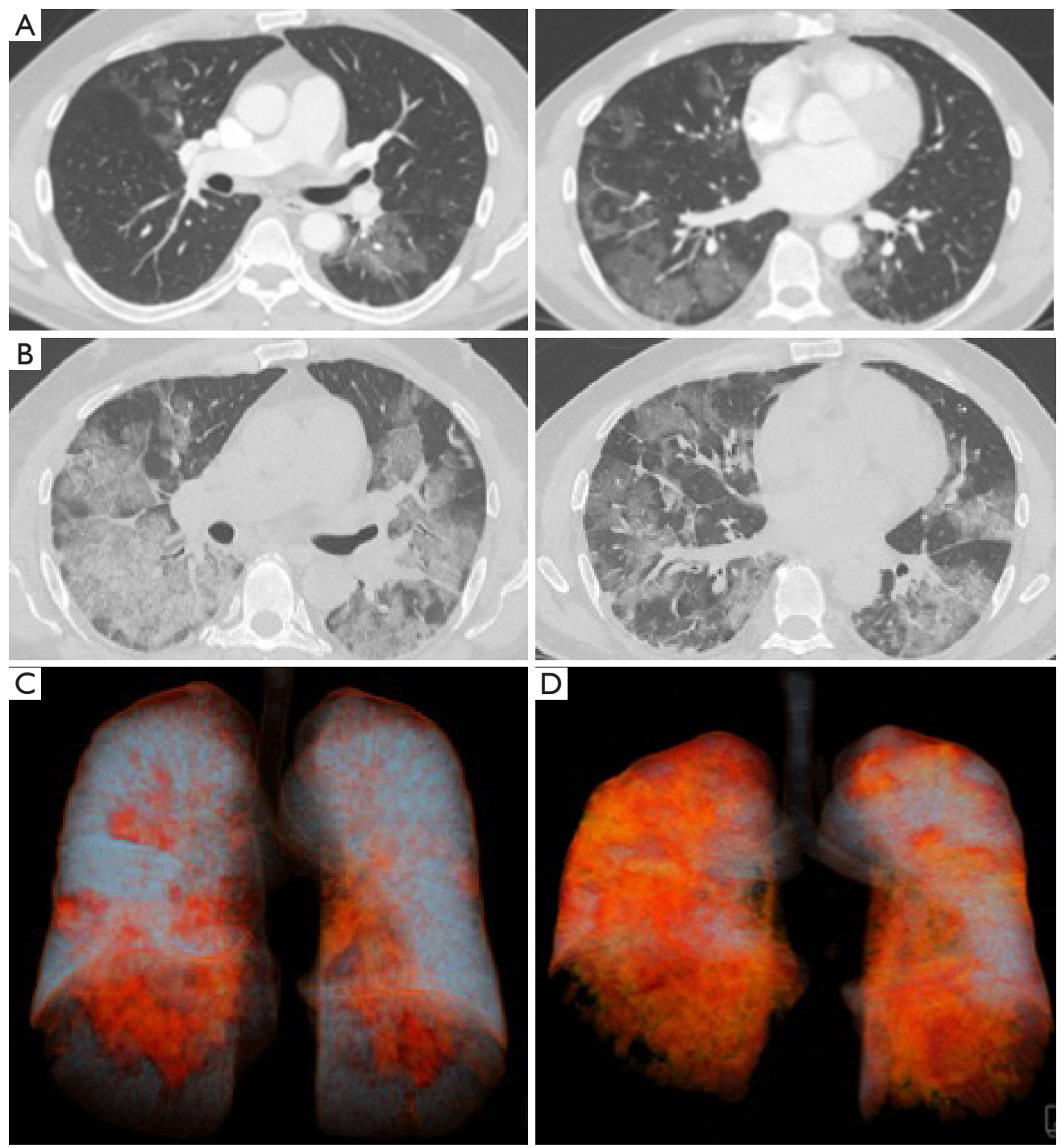

Figure 4 CT-Thorax findings and 3D Volume rendering show a typical COVID-19 infection with increasing involvement of pulmonary parenchyma during hospital stay. (A) CT Thorax at hospital admission with patchy peripheral and peribronchovascular ground glass opacities displayed here in two sectional images. (B) CT Thorax 5 days after hospital admission. Compared to the initial CT, the involved parenchyma increased, the preexisting ground glass opacities got denser, new interlobular septal thickening is seen and a typical crazy paving pattern developed. Also new subpleural consolidations are seen. (C) 3-D Volume rendering visualizing the involved parenchyma in orange at hospital admission. (CT severity score 7/20). (D) 3-D Volume rendering visualizing the progressing involvement of lung parenchyma 5 days after hospital admission. (CT severity score 14/20). COVID-19, coronavirus disease 2019.

pneumonia/post-COVID fibrotic ILD is scheduled 6 and 12 months after discharge (October 2020, April 2021), with lung function tests and if required Chest-CT.

All procedures performed in studies involving human participants were in accordance with the ethical standards of the institutional research committee(s) and with the Helsinki Declaration (as revised in 2013). Written informed consent was obtained from the patient.

\section{Discussion}

We describe a typical SARS-CoV-2 radiological presentation in an immunocompromised patient but atypical clinic presentation, with triple negative nasopharyngeal swabs 
in the 96 hours following hospital admission despite a CT thorax highly suggestive for an early stage of infection, and COVID-19 infection confirmed by BAL, primary performed for exclusion of an alternative diagnosis. The initial respiratory symptoms were minimal, in comparison to gastrointestinal symptoms and persistent fever. Interestingly, BAL cytologic findings were consistent with an alveolar lymphocytosis. We believe our case is exemplary for many critical issues related to the current SARS-CoV2 pandemic. In addition, we describe novel cytologic findings of the BAL. First, our patient might have exemplary contributed to the rapid diffusion of COVID-19 infection over the globe. In our case, it's unclear where the patient was first infected. The incubation period of SARS-CoV2 ranges from 2 to 14 days with a median incubation period of 4 days (5). Our patient lives in the central part of Switzerland, a region of very low COVID-19 prevalence when he moved to USA. Due to the onset of the first symptoms, the COVID-19 infection was more probably acquired at the airport, during the overseas flight, or in the USA. No airport screening was performed at that time in the USA, and airport screening is reported as unlikely to detect a sufficient proportion of COVID-19 infected travellers to avoid entry of infected people (6). Second, our case describes a probably not uncommon respiratory pauci-symptomatic patient, with repeated negative COVID-19 swabs, but highly suggestive clinic and radiology signs of a SARS-CoV-2 disease. There is an increasing evidence for the diagnostic importance of radiology specially chest-CT in the SARS-CoV-2 diagnosis. Thorax-CT is superior to RT-PCR nasopharyngeal swaps for detecting SARS-CoV-2, with a sensitivity of $98 \%$ vs. $71 \%$ (7). The CT-finding allows a good differentiation between common viral pneumonia and COVID-19 pneumonia with $86 \%$ specificity (8). Moreover CT helps with the correlation between total CT severity score (involved lung parenchyma) and overall disease severity (3). To date, in order to establish a diagnosis naso- and oro-pharyngeal samples are usually collected from patients with suspected infection. If a first sample is negative, a second sample might be repeated after 24-48 hrs. However, as in our case the possibility of sequential false negative results has to be considered. This might be due to the sampling technique, but more likely in relation to the nature of the virus and his probable drift from the upper airway in an early phase of the disease to the lower airway in a late phase, after 6-8 days from symptoms onset. Currently, in our institution all COVID-19 suspected patient cases are isolated for at least 14 days after symptom onset and 48 hours after complete symptoms remission, regardless of a negative nasopharyngeal swab result. The isolation is extended up to 21 or 28 days in most severe cases (ICU-need and ventilation or tracheostomy-need), according the regularly updated guidelines of the Swiss national centre for infection and prevention (SWISSNOSO). The role of the BAL fluid in the diagnosis of a COVID-19 infection is debated (9). Accordingly to international recommendations (10) BAL sampling isn't indicated neither to confirm nor to rule-out a COVID-19 infection, mostly because of the staff exposure risk to a possible aerosolgeneration procedure such bronchoscopy (11) despite BAL fluid being more sensitive than other analyzes (93\% against $63 \%$ by nasal Swab) (12). In our case, we performed BAL to rule out an alternative diagnosis in an immunocompromised host, such as drug toxicity, alveolar hemorrhage, other viral infection or a P.jirovecii pneumonia. Due to the national restrictions, cytologic features of BAL in COVID-19 have not been described in the literature yet. Interestingly, in our case the BAL fluid showed no significant neutrophylia (3\%) but a significant lymphocytosis of $35 \%$ and a marked reduced CD4/CD8 ratio, a pattern usually found in hypersensitivity pneumonitis. The absence of CD20-positive B-lymphocytes was attributed to treatment with Rituximab. No morphologic signs of viral infections or of repair were present in our case. Less severe BAL lymphocytosis is reported in the literature, but with a normal CD4/CD8 T-cell ratio of 1.7 (13).

Due to restrictive use of BAL, cytologic features have seldom been described in SARS and MERS, and so far not in SARS-CoV-2. In MERS, BAL showed increased number of neutrophils and macrophages (14). Pulmonary pathology in SARS-CoV-2 has been described in autopsy studies, where a diffuse alveolar damage has been reported (15). However, features of diffuse alveolar damage such as an increased number of macrophages or desquamated cells were not present in our case. It can be argued that the features of DAD are only found in late stages of the disease, whereas our patient was not severely compromised at the time of the BAL. In addition, increased numbers of neutrophils may rather indicate a secondary bacterial infection and are not due to the COVID-2 infection itself. Therefore, further studies of the morphological changes in BAL in SARS-CoV-2 are required, especially with a correlation to the severity of the clinical findings. For the therapy of COVID several treatment options are considered and under evaluation, as chloroquine or HCQ, antiretroviral combination with lopinavir/ritonavir, macrolids antibiotics, tocilizumab and systemic corticosteroids (16). Chloroquine showed in in-vitro studies and in the animal models an 
antiviral activity against the SARS virus $(17,18)$ and avian influenza (19). At least four studies focused their attention on benefits from chloroquine/HCQ in COVID-19. Overall, the conclusions from these papers suggest marginal benefit from chloroquine/HCQ (20-23). However, it should be taken in account that there is a large heterogeneity regarding patient populations, inclusion criteria, drug dosages and, therefore, a conclusive statement about the use of chloroquine/HCQ is hard to achieve.

In our case, we decided to administer HCQ because of a possibly superior antiviral and prophylactic activity than chloroquine (24). However, according to the most recent data, currently we wouldn't administrate HCQ anymore (25). No antiretroviral combination lopinavir/ritonavir was administrated mainly because of the risk of interaction with co-medications and past therapy, but also because of recent studies in hospitalized adult patients with severe COVID-19 showing no clinical improvement or mortality with lopinavir-ritonavir treatment beyond standard care (24). However, treatment standards have to be reassessed based on current clinical trials. In addition, human immunoglobulin substitution with $\operatorname{IgG}$ was prescribed because of the baseline diagnosis and Rituximab medication, with secondary slightly reduced concentration of $\mathrm{IgG}$. There is a rationale for immunoglobulin supplementation in bacterial infections. The possible role of immunoglobuline supplementation is unclear and, at present, the US FDA has listed plasma as investigational new drug with a pending approval (26). Tocilizumab is a monoclonal antibody blocking the IL-6 receptor for IL-6 and consequently decreasing in the activity of the cytokine, which is centrally involved in the cytokine storm induced by COVID-19. Tocilizumab therapy has been suggested as an option in patients with high IL-6 levels, with bilateral prolonged pneumonia and with severe manifestations. To date, only two guidelines recommend the use of tocilizumab, the NHC and the SIMIT Lombardy section guidelines $(27,28)$. At day 5 we considered the administration of Tocilizumab, but we refrained because of the concomitant Rituximab therapy (also an exclusion criteria in most current studies). In absence of a co-medication with Rituximab, the patient would have fullfilled our current guidelines for the administration of Tocilizumab in SARS-CoV-2.

Our case report is exemplary for some of the pitfalls which may occur in a SARS-CoV-2 infections or in an immunocompromised patient. The initial atypical clinical presentation with gastrointestinal but almost no respiratory symptoms and the repeated negative nasopharyngeal swabs in an immunocompromised patient might have misled to another and wrong differential diagnoses. The respiratory symptoms occurred also in the course of the hospitalization might have been attributed to a nosocomial pneumonia. It was the beginning of the COVID-19 pandemic in Europe and no deep informations were available at that time. However, the early targeted use of Chest CTScan and the typical COVID-19 CT-changes in the lung parenchyma were crucial for a correct isolation of the patients and to guide the further investigations. The role of bronchoscopy in COVID-19 infections might be debated. We believe bronchoscopy is not primarily indicated to confirm a COVID-19 infections, if clinic and chest-CT are highly suspicious. However, bronchoscopy with adequate protective measures and BAL might have a role in all unclear cases, and to exclude other infections if suspected. In our case the BAL confirmed the SARS-CoV-2 infection. The founding of a BAL lymphocytosis with decreased CD4/CD8 T-cells ratio might have otherwise suggested an hypersensitivity pneumonitis, is firstly reported and requires further investigations.

\section{Conclusions}

Our case highlights the therapeutic challenges which have to be dealt with in patients with COVID-19 infection, who also suffer from significant co-morbidities affecting the immunsystem. Decision making should be guided by clinical and radiology presentations, it should supplant the results of diagnostic tests, particularly in cancer patients. A high clinical suspicion should supplant the false negative swab because early bronchoposcopic evaluation in cancer patients, who are receiving active treatment or are immunosuppressed, could allow the institution of the most effective treatment earlier.

\section{Acknowledgments}

Funding: None.

\section{Footnote}

Reporting Checklist: The authors have completed the CARE reporting checklist (available at http://dx.doi.org/10.21037/ atm-20-4307).

Conflicts of Interest: All authors have completed the ICMJE uniform disclosure form (available at http://dx.doi. 
org/10.21037/atm-20-4307). The authors have no conflicts of interest to declare.

Ethical Statement: The authors are accountable for all aspects of the work in ensuring that questions related to the accuracy or integrity of any part of the work are appropriately investigated and resolved. All procedures performed in studies involving human participants were in accordance with the ethical standards of the institutional research committee(s) and with the Helsinki Declaration (as revised in 2013). Written informed consent was obtained from the patient.

Open Access Statement: This is an Open Access article distributed in accordance with the Creative Commons Attribution-NonCommercial-NoDerivs 4.0 International License (CC BY-NC-ND 4.0), which permits the noncommercial replication and distribution of the article with the strict proviso that no changes or edits are made and the original work is properly cited (including links to both the formal publication through the relevant DOI and the license). See: https://creativecommons.org/licenses/by-nc-nd/4.0/.

\section{References}

1. Zhu N, Zhang D, Wang W, et al. A Novel Coronavirus from Patients with Pneumonia in China, 2019. N Engl J Med 2020;382:727-33.

2. British Society of Thoracic Imaging]. Available online: https://www.bsti.org.uk/

3. Li K, Yijie Fang Y, Wenjuan Li W, et al. CT image visual quantitative evaluation and clinical classification of coronavirus disease (COVID-19). Eur Radiol 2020;30:4407-16.

4. Liao X, Wang B, Kang Y. Novel coronavirus infection during the 2019-2020 epidemic: preparing intensive care units-the experience in Sichuan Province, China. Intensive Care Med 2020;46:357-60.

5. Guan WJ, Ni ZY, Hu Y, et al. Clinical Characteristics of Coronavirus Disease 2019 in China. N Engl J Med 2020;382:1708-20.

6. Quilty BJ, Clifford S, Flasche S, et al. Effectiveness of airport screening at detecting travellers infected with novel coronavirus (2019-nCoV). Euro Surveill 2020;25:2000080.

7. Fang Y, Zhang H, Xie J, et al. Sensitivity of Chest CT for COVID-19: Comparison to RT-PCR. Radiology 2020;296:E115-7.

8. Bai HX, Hsieh B, Xiong Z, et al. Performance of
Radiologists in Differentiating COVID-19 from NonCOVID-19 Viral Pneumonia at Chest CT. Radiology 2020;296:E46-54.

9. Ren LL, Wang YM, Wu ZQ, et al. Identification of a novel coronavirus causing severe pneumonia in human: a descriptive study. Chin Med J (Engl) 2020;133:1015-24.

10. <eref $>10$. Laboratory testing for 2019 novel coronavirus (2019-nCoV) in suspected human cases. Available at: https://www.who.int/publications-detailredirect/10665-331501</eref>

11. Group of Interventional Respiratory Medicine, Chinese Thoracic Society. Expert consensus for bronchoscopy during the epidemic of 2019 novel coronavirus infection (Trial version). Zhonghua Jie He He Hu Xi Za Zhi 2020;43:199-202.

12. Wang $W, \mathrm{Xu} Y$, Gao R, et al. Detection of SARSCoV-2 in Different Types of Clinical Specimens. JAMA 2020;323:1843-4.

13. Voiriot G, Fajac A, Lopinto J, et al. Bronchoalveolar lavage findings in severe COVID-19 pneumonia. Intern Emerg Med. 2020;15:1333-4.

14. Guery B, Poissy J, el Mansouf L, et al. Clinical features and viral diagnosis of two cases of infection with Middle East Respiratory Syndrome coronavirus: a report of nosocomial transmission. Lancet 2013;381:2265-72.

15. Barton LM, Duval EJ, Stroberg E, et al. COVID-19 Autopsies, Oklahoma, USA. Am J Clin Pathol 2020;153:725-33.

16. Xu X, Ong YK, Wang DY. Role of adjunctive treatment strategies in COVID-19 and a review of international and national clinical guidelines. Mil Med Res 2020;7:22.

17. Savarino A, Di Trani L, Donatelli I, et al. New insights into the antiviral effects of chloroquine. Lancet Infect Dis 2006;6:67-9.

18. Vincent MJ, Bergeron E, Benjannet S, et al. Chloroquine is a potent inhibitor of SARS coronavirus infection and spread. Virol J 2005;2:69.

19. Yan Y, Zou Z, Sun Y, et al. Anti-malaria drug chloroquine is highly effective in treating avian influenza A H5N1 virus infection in an animal model. Cell Res 2013;23:300-2.

20. Chloroquine diphosphate in two different dosages as adjunctive therapy of hospitalized patients with severe respiratory syndrome in the context of coronavirus (SARS-CoV-2) infection: Preliminary safety results of a randomized, double-blinded, phase IIb c... I medRxiv. Available online: https://www.medrxiv.org/content/10.110 $1 / 2020.04 .07 .20056424 \mathrm{v} 2$

21. Gautret P, Lagier JC, Parola P, et al. Hydroxychloroquine 
and azithromycin as a treatment of COVID-19: results of an open-label non-randomized clinical trial. Int J Antimicrob Agents 2020;56:105949.

22. Mahevas M, Tran VT, Roumier M, et al. No evidence of clinical efficacy of hydroxychloroquine in patients hospitalized for COVID-19 infection with oxygen requirement: results of a study using routinely collected data to emulate a target trial. medRxiv 2020. doi: 10.1101/2020.04.10.20060699.

23. Perinel S, Launay M, Botelho-Nevers É, et al. Towards Optimization of Hydroxychloroquine Dosing in Intensive Care Unit COVID-19 Patients. Clin Infect Dis 2020;71:2227-9.

24. Cao B, Wang Y, Wen D, et al. A Trial of LopinavirRitonavir in Adults Hospitalized with Severe Covid-19. N Engl J Med 2020;382:1787-99.

25. Mehra MR, Ruschitzka F, Patel AN. Retraction-

Cite this article as: Caci G, Minervini F, Fechner C, Roos JE, Obermann EC, Azzola A. Bronchoalveolar lavagedetected SARS-CoV-2 infection in presence of serial negative nasopharyngeal swabs: a case report. Ann Transl Med 2021;9(7):583. doi: 10.21037/atm-20-4307
Hydroxychloroquine or chloroquine with or without a macrolide for treatment of COVID-19: a multinational registry analysis. Lancet 2020;395:1820.

26. Research $\mathrm{C}$ for $\mathrm{BE}$ and. Investigational COVID-19 Convalescent Plasma [Internet]. U.S. Food and Drug Administration. FDA; 2020 [citato 22 ottobre 2020]. Available online: https://www.fda.gov/regulatoryinformation/search-fda-guidance-documents/ investigational-covid-19-convalescent-plasma

27. Chinese Clinical Guidance for COVID-19 Pneumonia Diagnosis and Treatment (7th edition). Available online: http://kjfy.meetingchina.org/msite/news/show/cn/3337.html

28. Lombardy Section Italian Society Infectious And Tropical Diseases. Vademecum for the treatment of people with COVID-19. Edition 2.0, 13 March 2020. Infez Med 2020;28:143-52. 